\title{
Beeping a Maximal Independent Set
}

\author{
Yehuda Afek · Noga Alon - Ziv Bar-Joseph · \\ Alejandro Cornejo - Bernhard Haeupler - Fabian Kuhn
}

\begin{abstract}
We consider the problem of computing a maximal independent set (MIS) in an extremely harsh broadcast model that relies only on carrier sensing. The model consists of an anonymous broadcast network in which nodes have no knowledge about the topology of the network or even an upper bound on its size. Furthermore, it is assumed that an adversary chooses at which time slot each node wakes up. At each time slot a node can either beep, that is, emit a signal, or be silent. At a particular time slot, beeping nodes receive no feedback, while silent nodes can only differentiate between none of its neighbors beeping, or at least one of its neighbors beeping.

We start by proving a lower bound that shows that in this model, it is not possible to locally converge to an MIS in sub-polynomial time. We then study four different relaxations of the model which allow us to circumvent the lower bound and find an MIS in polylogarithmic time. First, we show that if a polynomial upper bound on the network size is known, it is possible to find an MIS in $\mathcal{O}\left(\log ^{3} n\right)$ time. Second, if we assume sleep-
\end{abstract}

\section{Y. Afek}

The Blavatnik School of Computer Science,

Tel Aviv University, 69978, Israel

N. Alon

Sackler School of Mathematics,

Tel Aviv University, 69978, Israel

Z. Bar-Joseph

School of Computer Science,

Carnegie Mellon Univ., Pittsburgh, PA 15213, USA

\author{
A. Cornejo - B. Haeupler \\ Computer Science and Artificial Intelligence Laboratory, \\ Massachusetts Institute of Technology, MA 02139, USA \\ F. Kuhn \\ Department of Computer Science, \\ University of Freiburg, 79110 Freiburg, Germany
}

ing nodes are awoken by neighboring beeps, then we can also find an MIS in $\mathcal{O}\left(\log ^{3} n\right)$ time. Third, if in addition to this wakeup assumption we allow sender-side collision detection, that is, beeping nodes can distinguish whether at least one neighboring node is beeping concurrently or not, we can find an MIS in $\mathcal{O}\left(\log ^{2} n\right)$ time. Finally, if instead we endow nodes with synchronous clocks, it is also possible to find an MIS in $\mathcal{O}\left(\log ^{2} n\right)$ time.

Keywords Maximal Independent Set - Distributed . Beeps · Radio Networks · Asynchronous Wakeup

\section{Introduction}

An MIS is a maximal set of nodes in network such that no two nodes in the set are neighbors. Since the set is maximal every node in the network is either in the MIS or has a neighbor in the MIS. The problem of distributively finding an MIS has been extensively studied in various models [2, 5, 19, 11, 12, 10, 13, 16, 14, 23. and has many applications in networking, and in particular in radio sensor networks. Some of the practical applications include the construction of a backbone for wireless networks, as a foundation for routing and for clustering, and for generating spanning trees to reduce communication costs [19, 23].

This paper studies the problem of finding an MIS in the discrete beeping wireless network model introduced in [6]. The network is modeled as an undirected graph and time progresses in discrete and synchronous time slots. In each time slot a node can either transmit a "jamming" signal (called a beep) or detect whether at least one of its neighbors beeps. We believe that such a model is minimalistic enough to be implementable in many real world scenarios. For example, it can easily be implemented using carrier sensing alone, where 
nodes only differentiate between silence and the presence of a signal on the wireless channel. Further, it has been shown that such a minimal communication model is strong enough to efficiently solve non-trivial tasks [1, 6, 17, 22. The model is interesting from a practical point of view since carrier sensing typically uses less energy to communicate and reaches larger distances when compared with sending regular messages.

While this model is clearly useful for computer networks, it is also useful to model biological processes. In biological systems, cells communicate by secreting certain proteins that are sensed ("heard") by neighboring cells [5]. This is similar to a node in a radio network transmitting a carrier signal which is sensed ("heard") by its neighbors. Such physical message passing allows for an upper bound on message delay. Thus, for a computational model based on these biological systems, we can assume a set of synchronous and anonymous processes communicating using beeps [6] in an arbitrary topology. We have recently shown that a variant of MIS is solved by a biological process, sensory organ precursor (SOP) selection in flies, and that the fly's solution provides a novel algorithm for solving MIS [1]. Here we extend algorithms for this model in several ways as discussed below.

The paper has two parts. First we prove a lower bound that shows that in a beeping model with adversarial wake-up it is not possible to locally converge to an MIS in sub-polynomial time. Next we present several relaxations of this model under which polylogarithmic MIS constructions are possible.

The lower bound shows that if nodes are not endowed with any information about the underlying communication graph, and their wake-up time is under the control of the adversary, any (randomized) distributed algorithm to find an MIS requires at least $\Omega(\sqrt{n / \log n})$ rounds. We remark that this lower bound holds much more generally. We prove the lower bound for the significantly more powerful radio network model with collision detection and arbitrary message sizes. The lower bound is therefore not an artifact of the amount of information which can be communicated in the beeping model.

Following the lower bound, in the second part of the paper four weaker models are considered and a polylogarithmic time algorithm for an MIS construction is presented for each of these models. First, we present an algorithm that uses a polynomial upper bound on the size of the network, to compute an MIS in $\mathcal{O}\left(\log ^{3} n\right)$ rounds with high probability. Our next two algorithms assume that nodes are awakened by incoming beeps (wake-on-beep). First, we present an $\mathcal{O}\left(\log ^{2} n\right)$ rounds algorithm in the wake-on-beep model with sender col-
Table 1 Model restrictions and algorithmic running times

\begin{tabular}{c|l|c} 
Section & Assumptions & Running Time \\
\hline 4 & None (lower bound) & $\Omega(\sqrt{n / \log n})$ \\
5 & Upper bound on $n$ & $\mathcal{O}\left(\log ^{3} n\right)$ \\
6 & Wake-on-Beep + Sender & $\mathcal{O}\left(\log ^{2} n\right)$ \\
& Collision Detection & \\
7 & Wake-on-Beep & $\mathcal{O}\left(\log ^{3} n\right)$ \\
8 & Synchronous Clocks & $\mathcal{O}\left(\log ^{2} n\right)$
\end{tabular}

lision detection. Next, we present a $\mathcal{O}\left(\log ^{3} n\right)$ time algorithm that works without sender collision detection in the same wake-on-beep model. Finally, we show that even if nodes are only by an adversary (and not by incoming beeps) it is possible to use synchronous clocks to compute an MIS in $\mathcal{O}\left(\log ^{2} n\right)$ time without any information about the network. The results are summarized in Table 1. We highlight that all the upper bounds presented in this paper compute a stable MIS eventually and almost surely. That is, once an MIS is computed it is stable and the probability that no MIS is computed until time $t$ is exponentially small in $t$. Thus only the running times of our algorithms are randomized.

\section{Related Work}

The problem of finding an MIS has been recognized and studied as a fundamental distributed computing problem for a long time (e.g., [2, 3, 13, 18]). Perhaps the single most influential MIS algorithm is the elegant randomized algorithm of 2, 13], generally known as Luby's algorithm, which has a running time of $\mathcal{O}(\log n)$. This algorithm works in a standard message passing model, where nodes can concurrently and reliably send and receive messages over all point-to-point links to their neighbors. Métivier et al. [14] showed how to improve the bit complexity of Luby's algorithm to use only $\mathcal{O}(\log n)$ bits per channel $(\mathcal{O}(1)$ bits per round). For the case where the size of the largest independent set in the 2-neighborhood of each node is restricted to be a constant (known as bounded independence or growthbounded graphs), Schneider and Wattenhofer 21. presented an algorithm that computes an MIS in $\mathcal{O}\left(\log ^{*} n\right)$ rounds. This class of graphs includes unit disk graphs and other geometric graphs that have been studied in the context of wireless networks.

While several methods were suggested for computing an MIS in a distributed setting, most previous algorithms are designed for a classical message passing model without message interference and collisions and they are based on the assumption that nodes know something about the local or global topology of the network. The first effort to design a distributed MIS algorithm for a wireless communication model in which the number of neighbors is not known is by Moscibroda and 
Wattenhofer [15]. They provide an algorithm for the radio network model with a $\mathcal{O}\left(\log ^{9} n / \log \log n\right)$ running time. This was later improved [16] to $\mathcal{O}\left(\log ^{2} n\right)$. Both algorithms assume that the underlying graph is a unit disk graph (the algorithms also work for somewhat more general class of geometric graphs). In addition, while the algorithms solve the MIS problem in multi-hop networks with adversarial wake up, they assume that an upper bound on the number of nodes in the network is known. In addition to the upper bound assumption their model allows for (and their algorithm uses) messages whose size is a function of the number of nodes in the network.

The use of carrier sensing and collision detection in wireless networks has been studied in [4, 9, 22]. As shown in 22, collision detection can be powerful and can be used to improve the complexity of algorithms for various basic problems. Scheideler et al. 20] show how to approximate a minimum dominating set in a physical interference (SINR) model where in addition to sending messages, nodes can perform carrier sensing. In [8], it is demonstrated how to use carrier sensing as an elegant and efficient way for coordination in practice.

The present paper is not the first one that uses carrier sensing alone for distributed wireless network algorithms. A similar model to the beeping model considered here was first studied in [7, 17]. As used here, the model has been introduced in [6], where it is shown how to efficiently obtain a variant of graph coloring that can be used to schedule non-overlapping message transmissions. In [1] a variant of the beeping model, there called the fly model, was considered. The fly model makes three additional assumptions: that all the processes wake up at the same round, that a bound on the network size is known to the processes, and that senders can detect collisions. That is, processes can listen on the medium while broadcasting (as in some radio and local area networks). Apart from [1, the most closely related work to this paper are results from [22]. In 22], it is shown that in growth-bounded graphs (a.k.a. bounded independence graphs) an MIS can be computed in $\mathcal{O}(\log n)$ time using only carrier sensing. Specifically, they assume nodes have receiver-side collision detection, they know the polynomial growth function of the graph, they known an upper bound on the size of the network and they have unique identifiers. The present paper studies the MIS problem in general graphs under the beeping model.

\section{Model}

Following 6, we consider a synchronous communication network modeled by an arbitrary graph $G=(V, E)$ where the vertices $V$ represent processes and the edges represent pairs of processes that can hear each other. We denote the set of neighbors of node $u$ in $G$ by $N_{G}(u)=\{v \mid\{u, v\} \in E\}$. For a node $u \in V$ we use $d_{G}(u)=\left|N_{G}(u)\right|$ to denote its degree (number of neighbors) and we use $d_{\max }=\max _{u \in V} d_{G}(u)$ to denote the maximum degree of $G$.

Initially all processes are asleep, and a process starts participating in the round after it is woken up by an adversary. We denote by $G_{t} \subseteq G$ the subgraph induced by the processes which are participating in round $t$.

Instead of communicating by exchanging messages, we consider a more primitive communication model that relies entirely on carrier sensing. Specifically, in every round a participating process can choose to either beep or listen. If a process $v$ listens in round $t$ it can only distinguish between silence (i.e., no process $u \in N_{G_{t}}(v)$ beeps in round $t$ ) or the presence of one or more beeps (i.e., there exists at least one process $u \in N_{G_{t}}(v)$ that beeps in round $t$ ). Observe that a beep conveys less information than a conventional 1-bit message, for which it is possible to distinguish between no message, a message with a one, and a message with a zero.

Given an undirected graph $H$, a set of vertices $I \subseteq$ $V(H)$ is an independent set of $H$ if every edge $e \in$ $E(H)$ has at most one endpoint in $I$. An independent set $I \subseteq V(H)$ is a maximal independent set of $H$, if for all $v \in V(H) \backslash I$ the set $I \cup\{v\}$ is not independent.

An event is said to occur with high probability, if it occurs with probability at least $1-n^{-c}$ for any constant $c \geq 1$, where $n=|V|$ is the number of nodes in the underlying communication graph. For a positive integer $k \in \mathbb{N}$ we use $[k]$ as short hand notation for $\{1, \ldots, k\}$. In a slight abuse of this notation we use [0] to denote the empty set $\varnothing$ and for $a, b \in \mathbb{N}$ and $a<b$ we use $[a, b]$ to denote the set $\{a, \ldots, b\}$.

During the execution of an algorithm each node may go through several different states. Of particular interest are the inactive-state and the MIS-state, which are present in all the algorithms described in this paper. A node is defined as stable if it is in the MIS-state and all its neighbors are in the inactive-state, or if it has a stable neighbor in the MIS-state. Observe that by definition, if all nodes are stable then all nodes are either in the MIS-state or in the inactive-state. In all our algorithms, once a node becomes stable it remains stable thereafter, and moreover eventually all nodes become stable with probability one. We will prove that the algorithms we propose guarantee that with high probability nodes becomes stable quickly and the nodes which are in the MIS-state describe a maximal independent set.

Specifically we say that a (randomized) distributed algorithm the MIS problem in $T$ rounds if, when no additional nodes are woken up for $T$ rounds, the nodes 
which are in the MIS-state describe a stable MIS (with high probability). Furthermore, we require that eventually the nodes which are in the MIS-state describe a stable MIS with probability one. Additionally, we say an algorithm locally converges to an MIS in $T$ rounds, if any node (with high probability) irrevocably decides $T$ rounds (regardless of wakeups) whether to be in the MIS-state or not.

\section{Lower Bound for Uniform Algorithms}

In this section we show that without any additional power or a priori information about the network, e.g., an upper bound on its size or maximum degree, any randomized distributed algorithm that locally converges to an MIS needs at least polynomial time.

We stress that this lower bound is not an artifact of the beeping model, but a limitation that stems from having message transmission with collisions and the fact that nodes are required to decide (but not necessarily terminate) without waiting until all nodes have woken up (i.e., locally converge). Although we prove the lower bound for the problem of finding an MIS, the bound can be generalized to other problems (e.g., minimal dominating set, coloring, etc.).

Specifically, we prove the lower bound for the much stronger communication model of local message broadcast with collision detection. In this model a process can choose in every round either to listen or to broadcast a message (no restrictions are made on the size of the message). When listening a process receives silence if no message is broadcast by its neighbors, it receives a collision if a message is broadcast by two or more neighbors, and it receives a message if it is broadcast by exactly one of its neighbors. The beep communication model can be easily simulated by this model (instead of beeping send a 1 bit message, and when listening translate a collision or the reception of a message to hearing a beep) and hence the lower bound applies to the beeping model.

At its core, our lower bound argument relies on the observation that a node can learn essentially no information about the graph $G$ if after waking up, it always hears collisions or silence. It thus has to decide whether it remains silent or beeps within a constant number of rounds. More formally:

Proposition 4.1 Let $\mathcal{A}$ be an algorithm run by all nodes, and consider a fixed pattern $H \in\{\text { silent, collision }\}^{*}$. If after waking up a node $u$ hears $H(r)$ whenever it listens in round $r$, then there are two constants $\ell \geq 1$ and $p \in(0,1]$ that depend on only $\mathcal{A}$ and $H$ such that either a) $u$ remains listening indefinitely, or $\mathbf{b}) u$ listens for $\ell-1$ rounds and broadcasts in round $\ell$ with probability $p$.
Proof. We fix a node $u$ and let $p(r)$ be the probability with which node $u$ beeps in round $r$. Observe that $p(r)$ can only depend on $r$, what node $u$ heard up to round $r$, that is, $H[1 \ldots r]$ and its random coin flips. Therefore, given any algorithm, either $p(r)=0$ for all $r$ (and node $u$ remains silent forever), or $p(r)>0$ for some $r$, in which case we let $p=p(r)$ and $\ell=r$.

We now prove the main result of this section:

Theorem 4.1 If nodes have no a priori information about the graph $G$ then any distributed algorithm in the local message broadcast model with collision detection that locally converges to an MIS requires with constant probability at least $\Omega(\sqrt{n / \log n})$ rounds.

Proof. We fix any algorithm $\mathcal{A}$ and use Proposition 4.1 to split the analysis into three cases. In all cases we show that there is a family of graphs on which, with probability $1-o(1)$, algorithm $\mathcal{A}$ does not locally converge to an MIS if it is run for $o(\sqrt{n / \log n})$ rounds.

We first ask what happens with nodes running algorithm $\mathcal{A}$ that hear only silence after waking up. Proposition 4.1 implies that either nodes remain silent forever, or there are constants $\ell$ and $p$ such that nodes broadcast after $\ell$ rounds with probability $p$. In the first case, suppose nodes are in a clique, and observe that no node will ever broadcast anything. In this case nodes cannot learn anything about the underlying graph and in particular cannot break symmetry between them. Thus, either no node joins the MIS, or all nodes join the MIS independently with constant probability, in which case their success probability is exponentially small in $n$.

We can thus apply Proposition 4.1 and assume for the rest of the argument that nodes running $\mathcal{A}$ that hear only silence after waking up broadcast after $\ell$ rounds with probability $p$. Now we consider what happens with nodes running $\mathcal{A}$ that hear only collisions after waking up. Again, by Proposition 4.1 we know that either they remain silent forever, or there are constants $m$ and $p^{\prime}$ such that nodes broadcast after $m$ rounds with probability $p^{\prime}$. In the rest of the proof we describe a different execution for each of these cases.

\section{CASE 1: (a node that hears only collisions re- mains silent forever)}

We consider a network topology consisting of several interconnected node-disjoint cliques. For some $k \gg \ell$ to be fixed later, we take a set of $k-1$ cliques $C_{1}, \ldots, C_{k-1}$ and a set of $k$ cliques $U_{1}, \ldots, U_{k}$, where each clique $C_{i}$ has $\Theta(k \log n / p)$ vertices, and each clique $U_{j}$ has $\Theta(\log n)$ vertices. We consider a partition of each clique $C_{i}$ into $k$ sub-cliques $C_{i}(1), \ldots, C_{i}(k)$ where each subclique has $\Theta(\log n / p)$ vertices. For simplicity we say two 


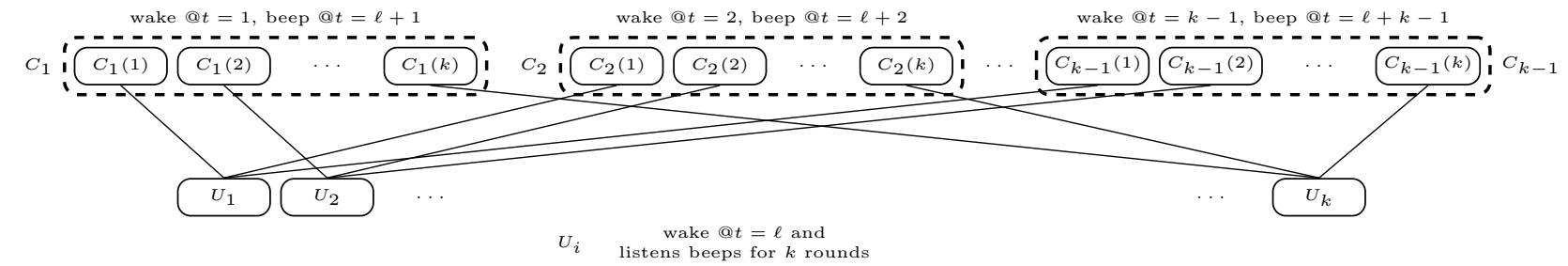

Fig. 1 Execution for Case 1

cliques are connected if they form a complete bipartite graph.

For every $j \in[k]$ clique $U_{j}$ is connected to sub-clique $C_{i}(j)$ for each $i \in[k-1]$. We consider the execution where in round $i \in[k-1]$ clique $C_{i}$ wakes up, and in round $\ell$ the cliques $U_{1}, \ldots, U_{k}$ wake up simultaneously. Hence, when clique $U_{j}$ wakes up, it is connected to subclique $C_{i}(j)$ for each $i<\ell$. Similarly for $i \geq \ell$, when clique $C_{i}$ wakes up, for all $j \in[k]$, sub-clique $C_{i}(j)$ is connected to clique $U_{j}$.

Because the first nodes wake up in round 1, no node participates in round 1 . During the rounds $2, \ldots, \ell$, only the nodes in $C$-cliques are participating and they all remain silent and hear silence. In round $\ell+1$ every node in $C_{1}$ broadcasts with probability $p$. Thus with high probability for all $j \in[k]$ at least two nodes in subclique $C_{1}(j)$ broadcast in round $\ell$. This guarantees that all the nodes in the $U$-cliques hear a collision during the first round they are awake, and hence they also listen for the second round. In turn, this implies that the nodes in $C_{2}$ hear silence during the first $\ell-1$ rounds they participate, and again for $j \in[k]$, with high probability, there are at least two nodes in $C_{2}(j)$ that broadcast in round $\ell+2$.

We can extend this argument inductively to show that, with high probability. For each $i \in[k-1]$ and for every $j \in[k]$ at least two nodes in sub-clique $C_{i}(j)$ broadcast in round $\ell+i$. Therefore, with high probability, all nodes in cliques $U_{1}, \ldots, U_{k}$ hear collisions during the first $k-1$ rounds after waking up.

Observe that at most one node in each $C_{i}$ clique can join the MIS, that is, only one of the sub-cliques of $C_{i}$ has a node in the MIS. Since there are more $U$-cliques than there are $C$-cliques the pigeon hole principle implies that there exists at least one clique $U_{j}$ that is connected to only non-MIS nodes. However, since the nodes in $U_{j}$ are connected in a clique, exactly one node of $U_{j}$ must decide to join the MIS. Note that all nodes in $U_{j}$ have the same state during the first $k-1$ rounds. Therefore, if nodes decide after participating for at most $k-1$ rounds, with constant probability, either no node in $U_{j}$ joins the MIS, or more than two nodes join the MIS.
Finally since the number of nodes $n$ is $\Theta\left(k^{2} \log n+\right.$ $k \log n)$, we can let $k \in \Theta(\sqrt{n / \log n})$ and the claim follows.

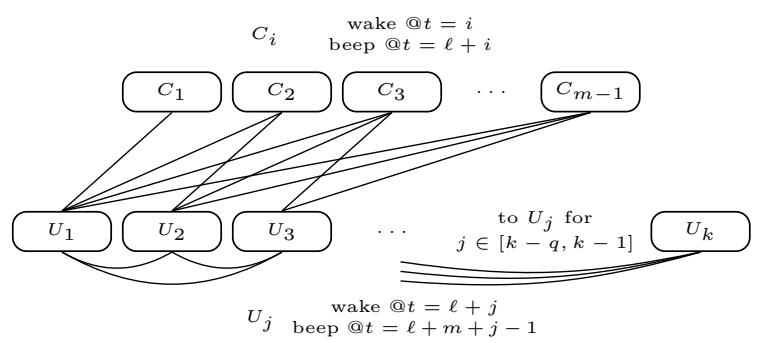

Fig. 2 Execution for Case 2

\section{CASE 2: (after hearing only collisions, a node beeps with probability $p^{\prime}$ after $m$ rounds)}

For some $k \gg m$ to be fixed later let $q=\left\lfloor\frac{k}{4}\right\rfloor$ and consider a set of $k$ cliques $U_{1}, \ldots, U_{k}$ and a set of $m-1$ cliques $C_{1}, \ldots, C_{m-1}$, where each clique $U_{i}$ is of size $\Theta\left(\log n / p^{\prime}\right)$, and each clique $C_{i}$ is of size $\Theta(\log n / p)$. As before, we say two cliques are connected if they form a complete bipartite graph.

If $j>1$ then $U_{j}$ is connected to every $U_{i}$ for $i \in$ $\{\max (1, j-q), \ldots, j-1\}$ and if $j<m$ then $U_{j}$ is connected to every clique $C_{h}$ for $h \in\{j, \ldots, m\}$. We consider the execution where in round $i \in[m-1]$ clique $C_{i}$ wakes up, and in round $\ell+j$ for $j \in[k]$ clique $U_{j}$ wakes up.

During the rounds $2, \ldots, \ell-1$, the nodes in $C_{1}$ are participating without hearing anything else, and hence every node in $C_{1}$ broadcasts in round $\ell+1$ with probability $p$. Therefore, with high probability, at least two nodes in $C_{1}$ broadcast in round $\ell+1$. This guarantees the nodes in $U_{1}$ hear a collision after waking up at round $\ell+1$, and therefore they listen in round $\ell+2$. In turn this implies the nodes in $C_{2}$ will also hear silence during the first $\ell-1$ rounds they participate, and hence, with high probability, at least two nodes in $C_{2}$ broadcast in round $\ell+2$.

As before, we can extend this execution inductively to show that for $i \in[m-1]$ the nodes in $C_{i}$ hear silence for the first $\ell-1$ rounds they participate, and, with 
high probability, at least two nodes in $C_{i}$ broadcast in round $\ell+i$. Moreover, for $j \in[k]$ the nodes in $U_{j}$ hear collisions for the first $m-1$ rounds they participate, and hence with high probability there are at least two nodes in $U_{j}$ who broadcast in round $\ell+m+j-1$. This implies that with high probability for $j \in[k-q]$ the nodes in $U_{j}$ hear collisions for the first $q$ rounds they participate.

We show that if nodes choose whether or not to join the MIS $q$ rounds after participating, then they fail with high probability. In particular consider the nodes in clique $U_{j}$ for $j \in\{q, \ldots, k-2 q\}$. These nodes will hear collisions for the first $q$ rounds they participate, and they are connected only to other nodes which also hear collisions for the first $q$ rounds they participate. Therefore, if nodes decide after participating for at most $q$ rounds, with constant probability either a node and all its neighbors will not be in the MIS, or two or more neighboring nodes join the MIS.

Finally since we have $n \in \Theta(m \log n+k \log n)$ nodes, we can let $k \in \Theta(n / \log n)$ and hence $q \in \Theta(n / \log n)$ and the theorem follows.

\subsection{Termination Lower Bound}

In this section we provide a basic observation about symmetry breaking in beep networks. We conclude that no MIS algorithm for this model can safely terminate at any time. This justifies why all our algorithms guarantee safety by running indefinitely.

We note that for the same reasons as before, the results in this subsection hold even for local message broadcast with collision detection. Moreover they hold under the assumptions that nodes wake up at the same time and know the size of the network. This includes the knowledge of an upper bound on the size of the network assumed in Section 5 , the wake-on-beep model in Section 7 and the assumption of synchronized clocks in Section 8 It applies thus to all our algorithms, except the one in Section 6 .

Lemma 4.1 It is impossible for a node to distinguish at time $t$ with probability more then $1-2^{-t+1}$ between an execution in which it is in isolation and an execution in which it has exactly one neighbor.

Proof. Initially nodes start in identical states and the probability of distinguishing between being isolated (1node graph) or having one neighbor (2-node graph) is at most $1 / 2$. In each round the symmetry between two neighboring nodes is broken only if one node beeps while the other node listens. Since the nodes are assumed to be in identical states they both have the same probability $p$ to beep. Therefore the probability that symmetry is broken for the first time in any particular round is $\max _{p} 2(1-p) p \leq 1 / 2$. Hence the probability that after $t$ rounds the nodes remain in identical states is at least $2^{-t}$. Finally this implies that the probability that after $t$ rounds a node cannot distinguish between an execution in which it is isolated and an execution where it has exactly one neighbor is at most $1-2^{-t+1}$.

Lemma 4.2 An algorithm that solves the MIS problem cannot terminate with a correct solution in every execution.

Proof. In a 1-node graph a node must join the MIS while in a connected 2-node graph exactly one node must join the MIS and the other must not. Therefore, a node cannot terminate if it cannot distinguish between these two cases. Finally, by Lemma 4.1 at any time $t$ there is a non-zero probability for any algorithm to not being able to distinguish between these two cases.

Lemma 4.3 There are graphs where the expected time for any algorithm to converge to a stable MIS is at least $\frac{\log n}{e}$.

Proof. Consider a graph of $n / 2$ disjoint pairs of neighboring nodes. A MIS algorithm can only terminate if it has broken the symmetry in each component where breaking the symmetry is independent between components. By Lemma 4.1 for any $t$ the probability for this to happen is at most $\left(1-2^{-t}\right)^{n / 2}$. Using Markov's inequality the expected time until all pairs break symmetry is at least $k\left(1-2^{-k}\right)^{n / 2}$ for any $k$. Setting $k=\log n$ shows that the expected time to compute an MIS is at least $\log n\left(1-\frac{1}{n}\right)^{n / 2}$ and for $n \geq 2$ this implies the expected time to compute an MIS is at least $\log n / e$.

All our algorithms will always (eventually) converge to a stable MIS, and with high probability they converge to an MIS in polylogarithmic time. Lemma 4.2 and Lemma 4.3 imply that both properties are best possible. A Las Vegas algorithm is one which always produces the correct output but whose running time is probabilistic. Conversely, a Monte Carlo algorithm is one whose running time is deterministic but only produces the correct output with high probability. As we pointed out, all the algorithms presented in this paper are Las Vegas. However by assuming an upper bound on $n$ it is possible to turn any of these algorithms into a Monte Carlo algorithm. Specifically, it suffices to add an early-stopping criteria (using the upper bound on $n$ ) once the output is correct with high probability. Another alternative to convert these Las Vegas algorithms to Monte Carlo is to endow nodes with unique identifiers. Specifically, using these identifiers it is possible to augment the algorithms to detect the case where two 
neighboring nodes are in the MIS state with certainty in asymptotically the same round complexity as the bit length of the identifiers. Yet another alternative to circumvent Lemma 4.1 is to assume sender-side collision detection, an assumption which we consider detail in Section 6. This allows two nodes to detect in a single round whether they are beeping alone, or if there is another neighbor beeping. Our algorithm in Section 6 leverages this assumption to terminate after $\mathcal{O}\left(\log ^{2} n\right)$ rounds.

\section{Using an Upper Bound on $n$}

In this section we give an example demonstrating that knowing a priori information about the network can drastically change the complexity of the problem. More precisely we show that by giving all nodes a (crude) upper bound $N>n$ on the total number of nodes participating in the system, it is possible to circumvent the polynomial lower bound for Section 4 and design an algorithm that locally converges to an MIS in polylog time. It is not required that all nodes are given the same upper bound. We will describe an algorithm that guarantees that $\mathcal{O}\left(\log ^{2} N \log n\right)$ rounds after a node wakes up, it knows whether it belongs to the MIS or if it has a neighbor in the MIS. This implies that if the known upper bound is polynomial in $n$ its possible to design an algorithm that locally converges to an MIS in $\mathcal{O}\left(\log ^{3} n\right)$ rounds.

\section{Algorithm.}

If at any point during the execution a node hears a beep while listening it restarts the algorithm. When a node wakes up (or it restarts), it stays in an inactive state where it listens for $c \log ^{2} N$ consecutive rounds. After this inactivity period, nodes enter a competing state where rounds are grouped into $\log N$ phases of $c \log N$ consecutive rounds. Observe that due to the adversarial wake up and the restarts, the phases of different nodes may not be synchronized. In each round of phase $i$, a node beeps with probability $2^{i} / 8 N$, and otherwise it listens. Therefore by phase $\log N$ a node beeps with constant probability in every round. After successfully going through the $\log N$ competing phases (recall that when a beep is heard during any phase, the algorithm restarts) a node assumes it has joined the MIS and goes into an infinite loop where it beeps half of the time to claim its MIS status while listening the rest of the time to detect if a neighboring node is also in the MIS.

Theorem 5.1 If $N$ is an upper bound on $n$ known to the nodes, Algorithm 1 locally converges to an MIS in $O\left(\log ^{2} N \log n\right)$ rounds.

We remark that Algorithm 1 is very robust. It is not hard to show that it is self-stabilizing, that is, nodes

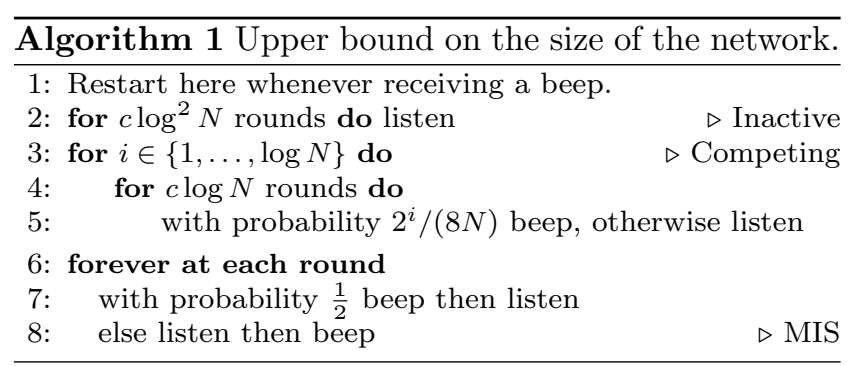

can be initialized in any state and with any setting of internal variables without affecting the guarantees. It also works as-is under adversarial crashes, that is, if we give the adversary the power to crash any set of nodes in every round. However, in the presence of crashes, no algorithm can locally converge to an MIS, since an inactive node with a single neighboring MIS node cannot always immediately join the MIS when its MIS neighbor crashes. Nevertheless, Algorithm 1 computes an MIS in $O\left(\log ^{2} N \log n\right)$ rounds. We also refer to the discussion in Section 4.1 which shows that without additional assumptions this Las Vegas algorithm can be turned into a Monte Carlo algorithm that with high probability gives a correct answer and always terminates in $O\left(\log ^{3} N\right)$ steps.

\section{Safety.}

We first prove the safety property of Algorithm 1 in the following lemma:

Lemma 5.1 Two neighboring nodes do not join the MIS with high probability. Moreover, in the low probability event that two neighboring nodes join the MIS, then almost surely eventually one of them becomes inactive.

Proof. Observe that a node must go through an interval of at least $c \log N$ rounds in which it is both listening and beeping with constant probability in every round. If a node is competing while another node is in the MIS or if two nodes are competing for the MIS in their last phase both nodes need to choose the same action (beep or listen) for $c \log N$ rounds in order for both nodes to be in the MIS state simultaneously. Therefore, for a sufficiently large constant $c$ this event will not happen with high probability. On the other hand, even if two neighboring nodes join the MIS, the probability that they both remain in the MIS after $k$ rounds is exponentially small in $k$, so it follows that eventually almost surely one of the nodes will leave the MIS.

We note that by construction nodes which are in the MIS beep at least every three rounds. Hence, if a node is in the MIS and all its neighbors are inactive, it follows that the MIS node and its neighbors will remain stable indefinitely (or until a neighbor crashes). 


\section{Termination.}

Given Lemma 5.1 it remains to show the following lemma to finish the proof of Theorem 5.1 .

Lemma 5.2 With high probability after $\mathcal{O}\left(\log ^{2} N \log n\right)$ rounds a node is either in the MIS or has a neighbor in the MIS.

We prove this in three steps. First we show that for any node, the sum of the beep probabilities of its neighbors cannot increase "quickly" after $c \log N$ rounds. We then use this to to show that when a node $u$ is competing, then with constant probability the sum of the beep probabilities of the neighbors of $u$ are less than a constant. Finally, we show that a node $u$ hears a beep or produces a beep every $O\left(\log ^{2} N\right)$ rounds. Every time this happens there is a constant probability that either a neighbor of $u$ joins the MIS or that $u$ joins the MIS. Therefore, with high probability the algorithm produces an MIS after $O\left(\log ^{2} N \log n\right)$ rounds.

First we introduce some additional definitions. We use $b_{u}(t)$ to denote the beep probability of node $u$ in round $t$. The beep potential of a set of nodes $S \subseteq V$ in round $t$ is defined as the sum of the beep probabilities of nodes in $S$ in round $t$, and denoted by $E_{S}(t)=$ $\sum_{u \in S} b_{u}(t)$. Of particular interest is the beep potential of the neighborhood of a node, we will use $E_{v}(t)$ as a shorthand notation for $E_{N(v)}(t)$.

The next lemma shows that if the beep potential of a particular set of nodes is larger than a (sufficiently large) constant in a particular round, then it was larger than a constant in the preceeding $c \log N$ rounds. Informally, this is true because the beep probability of every node increases slowly.

Lemma 5.3 Fix a set $S \subseteq V$. If $E_{S}(t) \geq \lambda$ in round $t$, then $E_{S}\left(t^{\prime}\right) \geq \frac{1}{2} \lambda-\frac{1}{8}$ for all $t^{\prime} \in[t-c \log N, t]$.

Proof. First we define a partition of the nodes in $S$. Let $P \subseteq S$ be the nodes in $S$ that are in phase 1 at round $t$, let $Q$ be the set of nodes which are in phase $i>1$ at round $t$, and let $R$ be the remaining nodes (i.e., the ones which are not competing). By definition the nodes in $R$ do not contribute to the beep potential of the nodes in $S$, we have:

$$
E_{S}(t)=\underbrace{\sum_{u \in P} b_{u}(t)}_{E_{P}(t)}+\underbrace{\sum_{u \in Q} b_{u}(t)}_{E_{Q}(t)}
$$

Fix $t^{\prime}$ to be any round in the range $[t-c \log N, t]$. Since nodes in $P$ are in phase 1 in round $t$, in round $t^{\prime}$ they are either in the inactive state or in phase 1. Thus for $u \in P$ we have $b_{u}\left(t^{\prime}\right) \leq b_{u}(t)=1 /(4 N)$, and since there are at most $|P| \leq|S| \leq N$ nodes, we have $E_{P}\left(t^{\prime}\right) \leq E_{P}(t)=(N / 4) N=1 / 4$.

Similarly nodes in $Q$ are in phase $i>1$ in round $t$ and in phase $i$ or $i-1 \geq 1$ in round $t^{\prime}$. Thus for $u \in Q$ we have $b_{u}\left(t^{\prime}\right) \geq \frac{1}{2} b_{u}(t)$ and hence $E_{Q}\left(t^{\prime}\right) \geq$ $\frac{1}{2} E_{Q}(t)=\frac{1}{2}\left(E_{S}(t)-E_{P}(t)\right) \geq \frac{1}{2} \lambda-\frac{1}{8}$. Finally since $E_{S}\left(t^{\prime}\right) \geq E_{Q}\left(t^{\prime}\right)$, we get $E_{S}\left(t^{\prime}\right) \geq \frac{1}{2} \lambda-\frac{1}{8}$.

Using the previous lemma, we show that with high probability nodes that are competing have neighborhoods with a "low" beep potential. Informally this is because if a node had a neighborhood with a "high" beep potential, the previous result implies it would have had a high beep potential during the previous $c \log N$ rounds, and therefore with high probability it would have been kicked out of the competition in a previous round.

Lemma 5.4 With high probability, if node $v$ is competing in round then $E_{v}(t)<\frac{1}{2}$.

Proof. Fix a node $v$ and a time $t$, we will show that if $E_{v}(t) \geq \frac{1}{2}$ then with high probability node $v$ is not competing at time $t$.

Let $L_{v}(\tau)$ be the event that node $v$ listens in round $\tau$ and there is a neighbor $u \in N(v)$ that beeps in round $\tau$. First we estimate the probability of the event $L_{v}(\tau)$.

$$
\begin{aligned}
\operatorname{Pr}\left[L_{v}(\tau)\right] & =\left(1-b_{v}(\tau)\right) \cdot\left(1-\prod_{u \in N(v)}\left(1-b_{u}(\tau)\right)\right) \\
& \geq\left(1-b_{v}(\tau)\right) \cdot\left(1-\exp \left(-\sum_{u \in N(v)} b_{u}(\tau)\right)\right) \\
& =\left(1-b_{v}(\tau)\right) \cdot\left(1-e^{-E_{v}(\tau)}\right)
\end{aligned}
$$

From Lemma 5.3 we have that if $E_{v}(t) \geq \frac{1}{2}$ then $E_{v}(\tau) \geq \frac{1}{8}$ for $\tau \in[t-c \log N, t]$, together with the fact that $b_{v}(\tau) \leq \frac{1}{2}$ this implies that

$\operatorname{Pr}\left[L_{v}(\tau)\right] \geq \frac{1}{2}\left(1-e^{-1 / 8}\right)>0.058$

for $\tau \in[t-c \log N, t]$.

Let $C_{v}(t)$ be the event that node $v$ is competing in round $t$. Observe that if $L_{v}(\tau)$ occurs for $\tau \in[t-$ $c \log N, t]$ then node $v$ stops competing for at least $c \log N$ rounds and hence $C_{v}(t)$ cannot occur. Therefore, the probability that node $v$ does not beep in round $t$ is at least: 
$\operatorname{Pr}\left[\neg C_{v}(t)\right] \geq \operatorname{Pr}\left[\exists \tau \in[t-c \log N, t]\right.$ s.t. $L_{v}(\tau)$ occurs $]$

$$
\begin{aligned}
& \geq 1-\prod_{\tau=t-c \log N}^{t}\left(1-\operatorname{Pr}\left[L_{v}(\tau)\right]\right) \\
& \geq 1-\exp \left(-\sum_{\tau=t-c \log N}^{t} \operatorname{Pr}\left[L_{v}(\tau)\right]\right) .
\end{aligned}
$$

Finally since for $\tau \in[t-c \log N, t]$, it holds that $\operatorname{Pr}\left[L_{v}(\tau)\right]>0.058$, then for a sufficiently large $c$ we have that node $v$ is not competing in round $t$ with high probability.

Next, we show that if a node hears a beep or produces a beep in a round when its neighborhood (and its neighbor's neighborhoods) has a "low" beep potential, then with constant probability either it joins the MIS, or one of its neighbors joins the MIS. In the following lemma we say a node beeps alone at time $t$, if that node beeped at time $t$ and all of its neighbors listened at time $t$.

Lemma 5.5 Assume that node $v$ beeps or hears a beep in round $t$ and that $E_{u}(t) \leq \frac{1}{2}$ for every $u \in N(v) \cup\{v\}$. Then with probability at least $\frac{1}{e}$ either $v$ beeps alone, or one of its neighbors beeps alone in round $t$.

Proof. For simplicity we rename the set $N(v) \cup\{v\}$ to the set $\{1, \ldots, k\}$ where $k=|N(v)|+1$. For $i \in[k]$ we consider three events.

$A_{i}$ : Node $i$ beeps in round $t$.

$B_{i}$ : Node $i$ beeps alone in round $t$.

$$
S: \bigcup_{i \in[k]} B_{i}
$$

Our aim is to show that the event $S$ happens with constant probability. Fix $i \in[k]$, as a first step we show that $\operatorname{Pr}\left[B_{i} \mid A_{i}\right]$ is constant.

$$
\begin{aligned}
\operatorname{Pr}\left[B_{i} \mid A_{i}\right] & =\operatorname{Pr}\left[\overline{\bigcup_{w \in N(i)} A_{w}}\right]=\operatorname{Pr}\left[\bigcap_{w \in N(i)} \overline{A_{w}}\right] \\
& =\prod_{w \in N(i)}\left(1-b_{w}(t)\right) \\
& \geq \exp \left(-2 \sum_{w \in N(i)} b_{w}(t)\right)=e^{-2 E_{i}(t)}
\end{aligned}
$$

Moreover, since by assumption $E_{i}(t) \leq \frac{1}{2}$, it follows that $\operatorname{Pr}\left[B_{i} \mid A_{i}\right] \geq \frac{1}{e}$.
We define the following finite partition of the probability space:

$$
\begin{aligned}
& \xi_{1}=A_{1}, \\
& \xi_{2}=A_{2} \cap \neg A_{1}, \\
& \xi_{3}=A_{3} \cap \neg A_{2} \cap \neg A_{1}, \\
& \ldots \\
& \xi_{k}=A_{k} \cap \bigcap_{i=1}^{k-1} \neg A_{i} .
\end{aligned}
$$

Recall that by assumption our probability space is conditioned on the event that "node $v$ beeps or hears a beep in round $t$ ", or in other words $\exists i \in[k]$ such that $A_{i}$ has occurred. Moreover, observe that $\bigcup_{i=1}^{k} \xi_{i}=\bigcup_{i=1}^{k} A_{i}$, and thus $\operatorname{Pr}\left[\bigcup_{i=1}^{k} \xi_{i}\right]=1$.

Since the events $\xi_{1}, \ldots, \xi_{k}$ are pairwise disjoint, by the law of total probability we have:

$$
\operatorname{Pr}[S]=\sum_{i=1}^{k} \operatorname{Pr}\left[S \mid \xi_{i}\right] \operatorname{Pr}\left[\xi_{i}\right]
$$

Finally since $\operatorname{Pr}\left[S \mid \xi_{i}\right] \geq \operatorname{Pr}\left[B_{i} \mid \xi_{i}\right] \geq \operatorname{Pr}\left[B_{i} \mid A_{i}\right] \geq \frac{1}{e}$ then $\operatorname{Pr}[S] \geq \frac{1}{e} \sum_{i=1}^{k} \operatorname{Pr}\left[\xi_{i}\right]=\frac{1}{e}$.

The three previous lemmas give us the ingredients necessary to prove Lemma 5.2 and thus complete the proof of Theorem 5.1.

Proof of Lemma 5.2 We say a node has an event in round $t$, if it beeps or hears a beep in round $t$. First we claim that a node has an event every $\mathcal{O}\left(\log ^{2} N\right)$ rounds. Consider a node that does not hear a beep within $\mathcal{O}\left(\log ^{2} N\right)$ rounds (if it does hear a beep, the claim clearly holds). Then after $\mathcal{O}\left(\log ^{2} N\right)$ it will join the MIS and beep and the claim follows.

From Lemma 5.4 we know that when a node decides to beep, with high probability the beep potential of its neighborhood is less than $\frac{1}{2}$. We can use a union bound to say that when a node hears a beep, with high probability the beep was produced by a node with a beep potential less than $\frac{1}{2}$. Therefore, we can apply Lemma 5.5 to say that with constant probability every time a node has an event, either the node joins the MIS (if it was not in the MIS already) or it becomes covered by an MIS node.

Hence, with high probability after $\mathcal{O}(\log n)$ events, a node is either part of the MIS or it becomes covered by an MIS node. Since there is an event every $\mathcal{O}\left(\log ^{2} N\right)$ rounds, this implies that with high probability a node is either inside the MIS or has a neighbor in the MIS after $\mathcal{O}\left(\log ^{2} N \log n\right)$ rounds.

This completes the proof for Theorem 5.1 . 


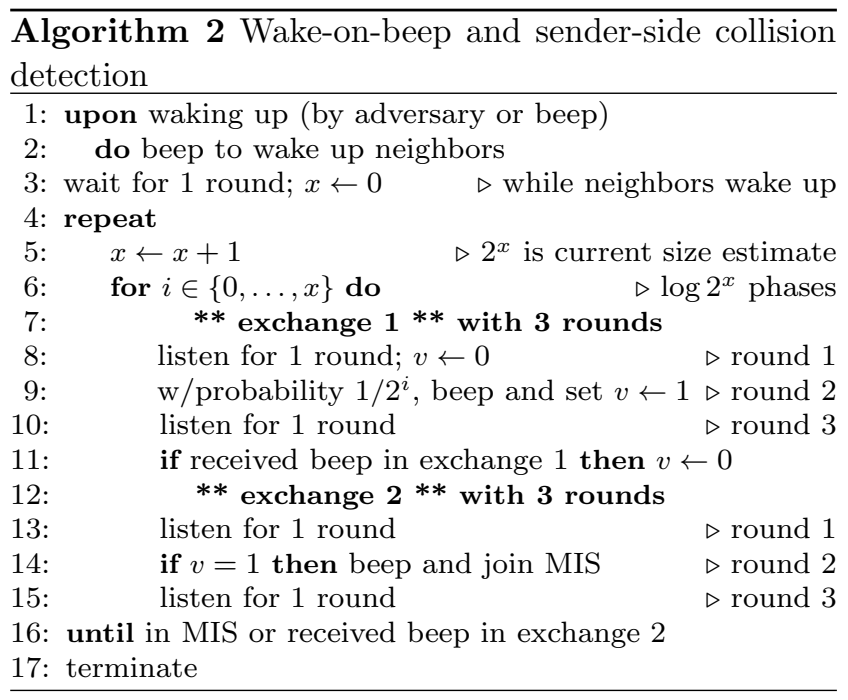

\section{Wake-on-Beep and Sender-Side Collision Detection}

This section considers a different relaxation of the beeping model. Specifically, while still allowing the adversary to wake up nodes arbitrarily, in this and the next section we assume that sleeping nodes also wake up upon receiving a beep. We call this the wake-on-beep assumption. Moreover, in this section we also assume that when a node beeps, it receives some feedback from which it can infer if it beeped alone, or if one of its neighbors beeped concurrently. We call this sender-side collision detection. We will show that in the wake-onbeep model with sender-side collision detection it is possible to locally converge to an MIS in $O\left(\log ^{2} n\right)$ time, even if nodes have no knowledge about the network topology, including its size.

This algorithm is an improvement of the algorithm presented in [1], which used an upper bound on the size of the network. In this algorithm nodes go through several iterations in which they gradually decrease the probability of being selected. The running time of the algorithm is still $O\left(\log ^{2} n\right)$ as we show below. Compared to the algorithm in [1, in addition to eliminating the dependence on any topological information, the current algorithm tolerates adversarial wake ups if we assume wake-on-beep.

\section{Algorithm.}

The algorithm proceeds in phases each consisting of $x$ steps where $x$ is the total number of phases performed so far (the phase counter). Step $i$ of each phase consists of two exchanges. In the first exchange nodes beep with probability $p_{i}$ (the value of $p_{i}$ is given by the algorithm), and in the second exchange a node that beeped in the first exchange and did not hear a beep from any of its neighbors, beeps again, signaling its neighbors it has joined the MIS and they should become inactive and exit the algorithm.

Nodes that are woken up by the adversary propagate a wave of wake-up beeps throughout the network. Upon hearing the first beep, which must be the wake up beep, a node broadcasts the wake up beep in the next round, and then waits one round to ensure none of its neighbors are still asleep. This ensures that all neighbors of a node wake up either in the same round as that node or one round before or after that node. Due to these possible differences in wakeup time, we divide each exchange into 3 rounds. During the second round of the first exchange each active node beeps with probability $p_{i}$ (the value of $p_{i}$ is given in the algorithm). The second exchange also takes three rounds. A node that beeps in the first exchange joins the MIS if none of its neighbors beeped in any of the three rounds of the first exchange. Such a node again beeps in the second round of the second exchange signaling its neighbors to terminate the algorithm. The algorithm is detailed in Algorithm 2,

\section{Safety.}

While the algorithm in [1 uses a different set of coin flip probabilities, it relies on a similar two exchanges structure to guarantee the safety properties of the algorithm. In 11, each exchange is only one round (since synchronous wakeup is assumed). We thus need to show that replacing each one round exchange with a three round exchange does not affect the MIS safety properties of [1]. We start by proving that the termination lemma from [1, which relies on the fact that all neighbors are using the same probability distribution in each exchange, still holds.

Lemma 6.1 All messages received by node $j$ in the first exchange of step $i$ were sent by processes using the same probability as $j$ in that step.

Proof. Let $k$ be a neighbor of $j$. If $k$ started in the same round as $j$ (both woke up at the same round) then they are fully synchronized and we are done. If $k$ started before $j$ then the first message $k$ sent has awakened $j$. Thus, they are only one round apart in terms of execution. Any message sent by $k$ in the second round of the first exchange of step $i$ would be received by $j$ in the first round of that exchange. Similarly, if $k$ was awakened after $j$ it must have been a 1 round difference and $j$ would receive $k$ 's message of the first exchange of step $i$ (if $k$ decided to beep) in the third round of that exchange. Thus, all messages received by $j$ are from processes that are also in step $i$ and so all processes from which $j$ receives messages in that exchange are using the same probability distribution. 
A similar argument would show that all messages received in the second exchange of step $i$ are from processes that are in the second exchange of that step. Since our safety proof [1] only relies on the coherence of the exchange it still holds for this algorithm.

\section{Termination.}

After establishing the safety guarantees, we next prove that with high probability all nodes terminate the algorithm in $O\left(\log ^{2} n\right)$ time where $n$ is the number of nodes that participate in the algorithm. Let $d_{v}$ be the number of active neighbors of node $v$. We start with the following definition of [2]. A node $v$ is good if it has at least $d_{v} / 3$ active neighbors $u$, such that, $d_{u} \leq d_{v}$. An edge is good if at least one of its endpoints is a good node.

Lemma 6.2 (Lemma 4.4 from [2]) In every graph $G$ at least half of the edges are good. Thus, $\sum_{v \in \text { good }} d_{v} \geq$ $|E| / 2$.

Note that we need less than $O\left(\log ^{2} n\right)$ steps to reach $x \geq \log n$, since each phase $x \leq \log n$ has less than $\log n$ steps. When $x \geq \log n$, the first $\log n$ steps in each phase are using the probabilities: $1,1 / 2,1 / 4, \ldots, 2 / n, 1 / n$. Below we show that from round $x=\log n$, we need at most $O(\log n)$ more phases to guarantee that all processes terminate with high probability. We say an edge is deleted if one of its endpoints joins the MIS.

Lemma 6.3 In a phase (with more than $\log n$ steps) in expectation a constant fraction of the edges are deleted.

Proof. Fix a phase $j$, and fix a good node $v$. We claim that the expected number of edges incident to $v$ that are deleted in phase $j$ is $\Omega\left(d_{v}\right)$. To prove the claim assume that at the beginning of phase $j, 2^{k} \leq d_{v} \leq 2^{k+1}$ for some $0<k<\log n$. If when we reach step $i=k$ in phase $j$ at least $d_{v} / 20$ edges incident to $v$ were already removed we are done. Otherwise, at step $i$ there are still at least $d_{v} / 3-d_{v} / 20>d_{v} / 4 \geq 2^{k-2}$ neighbors $u$ of $v$ with $d_{u} \leq d_{v}$. Let $A$ be the event that node $v$ or a neighbor $u$ with $d_{u}<d_{v}$ beeps. Node $v$ and all its neighbors $u$ are flipping coins with probability $\frac{1}{2^{k}}$ at this step and thus the probability of $A$ occurring is:

$\operatorname{Pr}(A) \geq 1-\left(1-\frac{1}{2^{k}}\right)^{2^{k-2}} \geq 1-e^{-1 / 4}$.

On the other hand, all such nodes $u$, and $v$, have less than $2^{k+1}$ neighbors. Thus, the probability that a node from this set that beeps does not collide with any other node is:

$\operatorname{Pr}($ no collisions $) \geq\left(1-\frac{1}{2^{k}}\right)^{2^{k+1}} \geq 1 / e^{4}$.

Thus, in phase $j$ a node $v$ has probability of at least (1$\left.\frac{1}{e^{1 / 4}}\right) \frac{1}{e^{4}} \geq \frac{1}{2^{8}}$ to be removed. Thus, the probability that $v$ is removed in phase $j$ is $\Omega(1)$ and hence the expected number of edges incident with $v$ removed during this phase is $\Omega\left(d_{v}\right)$, which completes our claim.

Combining the previous claim with Lemma 6.2 then we can use linearity of expectation to show that the expected number of edges deleted in each phase is at least $\Omega\left(\sum_{v \in \text { good }} d_{v}\right)=\Omega(|E|)$.

With this lemma in place, we are ready to prove the main theorem of this section.

Theorem 6.1 Using sender-side collision detection and wake-on-beep, Algorithm 2 locally converges to an MIS in $O\left(\log ^{2} n\right)$ rounds.

Proof. Note that since the number of edges removed in a phase in a graph $(V, E)$ is clearly always at most $|E|$, the last lemma implies that for any given history, with probability at least $\Omega(1)$, the number of edges removed in a phase is at least a constant fraction of the number of edges that have not been deleted yet. Therefore there are two positive constants $p$ and $c$, so that the probability that in a phase at least a fraction $c$ of the number of remaining edges are deleted is at least $p$. Call a phase successful if at least a fraction $c$ of the remaining edges are deleted during the phase.

By the above reasoning, the probability of having at least $z$ successful phases among $m$ phases is at least the probability that a binomial random variable with parameters $m$ and $p$ is at least $z$. By the standard estimates for binomial distributions, and by the obvious fact that $O(\log |E| / c)=O(\log n)$, starting from $x=$ $\log n$ we need an additional $O(\log n)$ phases to finish the algorithm. Since each of these additional $O(\log n)$ phases consists of $O(\log n)$ steps, and since as discussed above until $x=\log n$ we have less than $O\left(\log ^{2} n\right)$ steps, the total running time of the algorithm is $O\left(\log ^{2} n\right)$.

\section{Wake-on-Beep Without Sender-Side Collision Detection}

In the previous section we assumed that nodes are endowed with sender-side collision detection and can thus tell whether one of their neighbors beeped even in rounds in which they beep. In this section we remove this assumption and present an algorithm for the wake-onbeep model that locally converges to an MIS without using sender-side collision detection.

\section{Algorithm.}

To extend Algorithm 2 to a model with no collision detection we increase the number of exchanges in each step from 2 to $c x$ where $c$ is a constant derived below and $x$ is the same as in Algorithm 2 and represents the current estimate of the network size. Each series of 


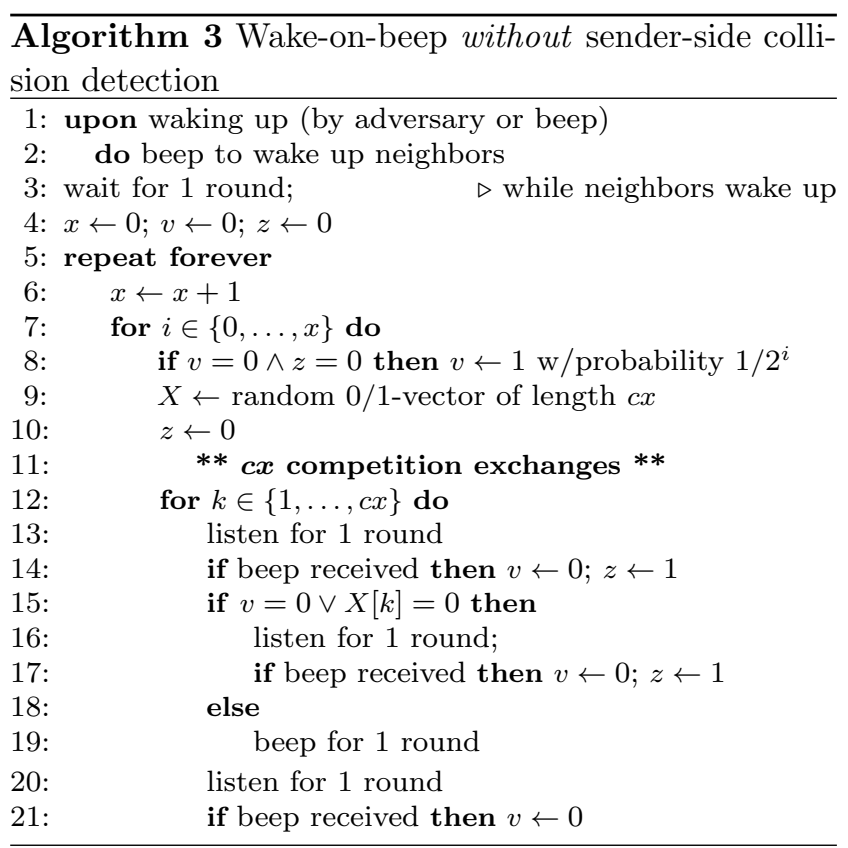

$c x$ rounds simulates with high probability an exchange with sender-side collision detection. Prior to starting the exchanges in each step each active process flips a coin with the same probability as in Algorithm 2. If the flip outcome is 0 (tails) the process only listens in the next $c x$ exchanges (for a constant $c$ discussed below). If the flip outcome is 1 the process sets $v=1$ and picks each entry in the vector $X$ of length $c x$ to be 1 or 0 independently and uniformly at random. Following this, the process picks one entry in the vector $X$ independently and uniformly at random and sets it to 1 (this is only to guarantee that at least one entry in $X$ is equal to one). In exchange $j$ of every phase, a process beeps if $X(j)=1$ and listens if $X(j)=0$. If at any of the exchanges it listens and hears a beep it sets $v=0$ and stops beeping (even in the selected exchanges). If a node hears a beep during these exchanges it does not exit the algorithm. Instead, it denotes the fact that one of its neighbors beeped and sets itself to be inactive. If it does not hear a beep in any of the exchanges of the following phase it becomes active and continues as described above. Similarly, a node that beeped and did not hear any beep in a specific step (indicating that it can join the MIS) continues to beep indefinitely (by selecting half the exchanges in all future steps to beep in them).

We say a process $u$ is in conflict with a neighbor $v$ if both have $v=1$. We say a process $u$ is in conflict if it is in conflict with respect to any of its neighbors.

The main difference between this algorithm and Algorithm 2 is the addition of a set of competition exchanges at the end of each coin flip. The number of competition exchanges is proportional to the current phase counter (which serves as the current estimate of the network size). Initially the competition rounds are short and so they would not necessarily remove all conflicts. We require that nodes that attempt to join continue to participate in all future competition rounds (when $v=1$ ). Processes that detect a MIS member as a neighbor set $z$ to 1 and do not beep until they go through one complete set of competition exchanges in which they do not hear any beep. If and when this happens they set $z=0$ and become potential MIS candidates again

While not all conflicts will be resolved at the early phases, when $x \geq \log n$ each set of competition exchanges is very likely to remove all conflicts. We prove below that once we arrive at such $x$ values, all conflicts are resolved with very high probability such that only one process in a set of conflicting processes remains with $v=1$ at the end of these competition exchanges. From there, it takes another $O(\log n)$ phases to select all members of the MIS as we have shown for Algorithm 1. Since each such phase takes $O(\log n)$ steps with each step taking $O(\log n)$ rounds for the competition, the total running time of the algorithm is $O\left(\log ^{3} n\right)$.

Lemma 7.1 Assume process $y$ is in conflict at step $i$ of phase $x \geq \log n$. The probability that $y$ remains in conflict at the end of the cx competition exchanges for step $i$ is at most $\frac{1}{n^{c / 3}}$.

Proof. If at any of the exchanges in this step all neighbors of $y$ have $v=0$ we are done. Otherwise in each exchange, with probability at least $1 / 4, y$ decided not to beep whereas one of its conflicting neighbors decided to beep. Thus, the probability that $y$ remains in conflict in a specific exchange is at most $3 / 4$. Since there are $(c \log n)$ exchanges in this step, the probability that $y$ is in conflict at the end of these exchanges is at most $\left(\frac{3}{4}\right)^{c \log n} \leq \frac{1}{n^{c / 3}}$.

Note that if two nodes remain in conflict after an exchange, they continue to beep in the following phase. As we proved in the previous section, if all conflicts are resolved in the $O(\log n)$ phases that follow the phase $x=\log n$ the algorithm will result in a MIS set with very high probability. Since we only need $O\left(\log ^{2} n\right)<n$ steps for this, and we have $n$ nodes, the probability that there exists a step and a node in phase $x \geq \log n$ such that a node that conflicted during this step with a neighbor does not resolve this conflict in that step is smaller than $\frac{1}{n^{c / 3-2}}$. Thus, with probability at least $1-\frac{1}{n^{c / 3-2}}$ all conflicts are resolved and the MIS safety condition holds.

We note that the fact that the vector $X$ always contains at least one 1 guarantees that once an MIS is com- 
puted it remains stable forever. We also remark that in contrast to the algorithm in Section 6, it is not possible for the algorithm in this section to terminate safely at any point of time (see Section 4.1 for details). This discussion completes the main proof of our main theorem for this section:

Theorem 7.1 In the wake-on-beep model, Algorithm 3 locally converges to an MIS in $O\left(\log ^{3} n\right)$ rounds.

\section{Synchronized Clocks}

For this section the only assumption we make on top of the beeping model is that that nodes have synchronized clocks, that is, know the current round number $t$.

The idea of the algorithm is to simulate Luby's permutation algorithm [13. In Luby's permutation algorithm a node picks a random $\mathcal{O}(\log n)$-size priority which it shares with its neighbors. A node then joins the MIS if it has the highest priority among its neighbors, and all neighbors of an MIS node become inactive. Despite the fact that we describe the algorithm for the message passing model, it is straightforward to adapt the priority comparisons to the beeping model. For this, a node sends its priority bit by bit, starting with the highestorder bit and using a beep for a 1. The only further modification is that a node stops sending its priority as soon as it hears a beep on a higher order bit during which it remained silent because it had a zero in the corresponding bit. Using this simple procedure, a node can easily realize when a neighboring node has a higher priority. Furthermore, nodes which do not hear any beep correspond to the nodes which have the highest-priority in its neighborhood (strictly speaking, this correspondence is not exact, since the algorithm described allows even more nodes to join the MIS that one step of Luby, but without violating any safety guarantees).

Therefore, as long as nodes have a synchronous start and know $n$ (or an upper bound on $n$ ) it is straightforward to get Luby's permutation algorithm working in the beeping model in $\mathcal{O}\left(\log ^{2} n\right)$ rounds.

In the rest of this section we show how to remove the need for an upper bound on $n$ and a synchronous start. We leverage synchronized clocks to synchronize the exchanges of priorities amongst neighboring nodes. Our algorithm keeps an estimate $k$ for the required priority-size $\mathcal{O}(\log n)$. Whenever two nodes tie for the highest priority the algorithm concludes that $k$ is not large enough and doubles its estimate. The algorithm uses a Restart-Bit to ensure that nodes locally work with the same estimate $k$ and run in a synchronized manner in which priority comparisons start at the same time (namely every $t \equiv 0(\bmod k))$. It is not obvious that either a similar $k$ or a synchronized priority comparison is necessary but it turns out that algorithms without them can stall for a long time. In the first case this is because nodes with a too small $k$ repeatedly enter the MIS state simultaneously, while in the second case many asynchronously competing nodes (even with the same, large enough $k$ ) keep eliminating each other without one becoming dominant and transitioning into the MIS state.

\section{Algorithm:}

Nodes have three different internal states: inactive, competing, and MIS. Each node has an estimate $k$ on the priority-size that is monotone increasing during the execution of the algorithm. Initially all nodes are in the inactive state with $k=6$.

Nodes communicate in beep-triplets, and synchronize by starting a triplet only when $t \equiv 0(\bmod 3)$. The first bit of the triplet is the Restart-Bit. A beep is sent in the Restart-Bit if and only if $t \not \equiv 0(\bmod k)$, otherwise a node listens in the Restart-Bit. If a node hears a beep in its Restart-Bit it doubles its estimate for $k$ and it becomes inactive. The second bit sent in the triplet is the MIS-Bit. A beep is sent for the MISBit if and only if a node is in the MIS state. If a node hears a beep on the MIS-bit it becomes inactive. The last bit sent in the triplet is the Competing-Bit. If inactive, a node listens in the Competing-Bit. If a node is competing it sends a beep with probability $1 / 2$ in the Competing-Bit. If a node is in the MIS state and it listened in the previous Competing-Bit then it beeps in the current Competing-Bit. On the other hand if node in the MIS state beeped in the previous Competing-Bit, then it flips a coin to decide weather to beep or listen in the current Competing-Bit. This ensures a node in the MIS state beeps every 2 round. If a node hears a beep on the Competing-Bit it becomes inactive, and if the node was in the MIS-state it also doubles its estimate for $k$. Lastly, a node transitions from inactive to competing (or from competing to MIS) between any time $t$ and $t+1$ for $t \equiv 0(\bmod k)$. The pseudo code is described in more detail in Algorithm 4.

\section{Analysis:}

The main result of this section is the following theorem.

Theorem 8.1 If nodes have synchronous clocks then Algorithm 4 solves the MIS problem in $\mathcal{O}\left(\log ^{2} n\right)$ rounds.

First, we show that with high probability $k$ cannot become super-logarithmic.

Lemma 8.1 With high probability $k \in \mathcal{O}(\log n)$ for all nodes during the execution of the algorithm.

Proof. We start by showing that two neighboring nodes in the MIS state must have the same estimate $k$ and 


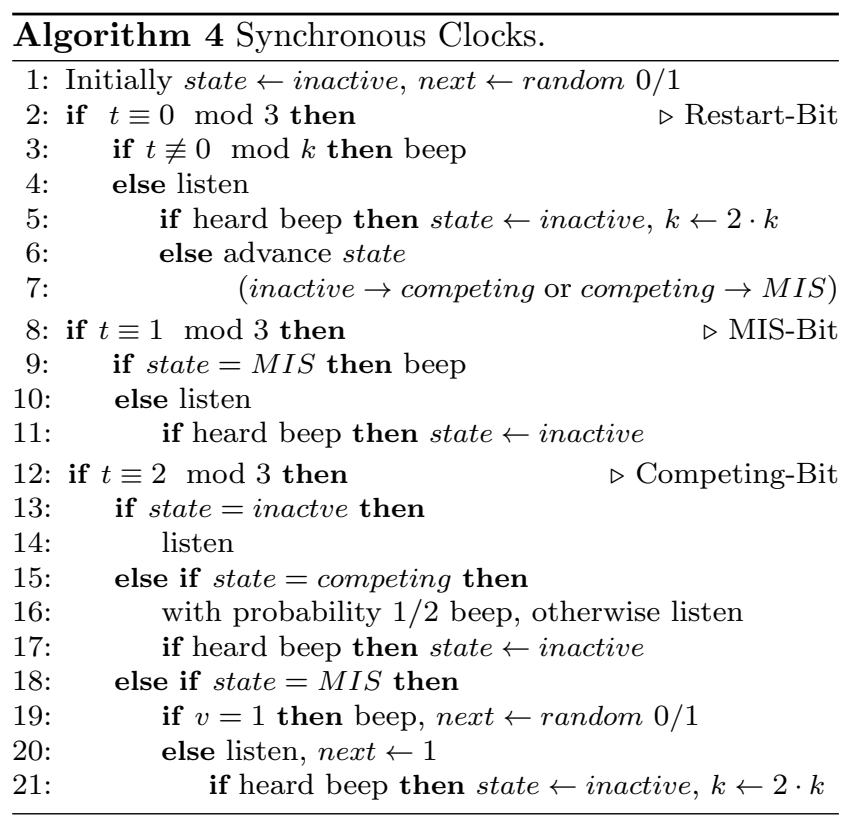

must have transitioned to the MIS state at the same time. We prove both parts of this statement by contradiction.

First, suppose by contradiction that two neighboring nodes $u$ and $v$ are in the MIS state but $u$ transitioned to this state (the last time) before $v$. In this case $v$ would have received the MIS-bit from $u$ and become inactive instead of joining the MIS - a contradiction.

Similarly, for sake of contradiction, now assume that the neighboring nodes $u$ and $v$ are in the MIS state and $k_{u}<k_{v}$. In this case, during the active phase of $u$ before it transitioned to the MIS at time $t$ it would have hear a beep in its Restart-Bit (produced by $v$ ) and would have switched to the inactive state, which contradicts that $u$ is in the MIS state.

We now use this to show that for a specific node $u$ it is unlikely to become the first node with a too large $k$. For this we note that $k_{u}$ is doubled because of a Restart-Bit only if a beep from a node with a larger $k$ is received. This node can therefore not be responsible for $u$ becoming the first node getting a too large $k$. The second way $k$ can increase is if a node transitions out of the MIS state because it receives a Competing-Bit from a neighbor $v$. In this case, we know that $u$ competed against at least one such neighbor for $k / 6$ phases without loosing in any of these phases. The probability that this happens is $2^{-k / 6}$. Hence, if $k \in \Theta(\log n)$, then with high probability it does not happen. A union bound over all nodes and the polynomial number of rounds in which nodes are not yet stable finishes the proof.

Lemma 8.2 If during an execution the $\mathcal{O}(\log n)$ neighborhood of node $u$ has not changed for $\Omega\left(\log ^{2} n\right)$ rounds then node $u$ is stable with high probability, i.e., $u$ is either in the MIS state with all its neighbors being inactive or it has at least one neighbor in the MIS state whose neighbors are all inactive.

Proof. First observe that if the whole graph has the same value of $k$ and no two neighboring nodes transition to the MIS state at the same time, then our algorithm behaves exactly as Luby's original permutation algorithm, and therefore terminates after $\mathcal{O}(k \log n)$ rounds with high probability. From a standard locality argument, it follows that a node $u$ also becomes stable if the above assumptions only hold for a $\mathcal{O}(k \log n)$ neighborhood around $u$. Moreover, since Luby's algorithm performs only $\mathcal{O}(\log n)$ rounds in the message passing model, we can improve our locality argument to show that in if a $\mathcal{O}(\log n)$ neighborhood around $u$ is wellbehaved, then $u$ behaves as in Luby's algorithm.

Since the values for $k$ are monotone increasing and propagate between two neighboring nodes $u$ and $v$ with different $k$ (i.e., $k_{u}>k_{v}$ ) in at most $2 k_{u}$ steps, it follows that for a node $u$ it takes at most $\mathcal{O}\left(k_{u} \log n\right)$ rounds until either $k_{u}$ increases or all nodes $v$ in the $\mathcal{O}(\log n)$ neighborhood of $u$ have $k_{v}=k_{u}=k$ for at least $\mathcal{O}(k \log n)$ rounds. We can furthermore assume that these $\mathcal{O}(k \log n)$ rounds are collision free (i.e, no two neighboring nodes go into the MIS), since any collision leads with high probability within $\mathcal{O}(\log n)$ rounds to an increased $k$ value for one of the nodes.

For any value of $k$, within $\mathcal{O}(k \log n)$ rounds a node thus either performs Luby's algorithm for $\mathcal{O}(\log n)$ priority exchanges, or it increases its $k$. Since $k$ increases in powers of two and, according to Lemma 8.1, with high probability it does not exceed $\mathcal{O}(\log n)$, after at most $\sum_{i}^{\mathcal{O}(\log \log n)} 2^{i} \cdot 3 \cdot \mathcal{O}(\log n) \in \mathcal{O}\left(\log ^{2} n\right)$ rounds the status labeling around a $\mathcal{O}(\log n)$ neighborhood of $u$ is a proper MIS. This means that $u$ is stable at some point, and the MIS-bit guarantees that no competing neighbor of $u$ will join the MIS and therefore stability is preserved for the rest of the execution.

We remark that as the algorithm of Section 5, this algorithm is also robust enough to work as-is with an adversary capable of crashing nodes (with the same caveats on the guarantees mentioned in Section 5).

Acknowledgements We thank the anonymous reviewers for their feedback to improve the quality of this paper. Research supported in part by AFOSR Award FA9550-08-1-0159, NSF Award CNS-1035199, NSF Award CCF-0937274, NSF Award CCF-0726514, ERC advanced grant, USA-Israeli BSF grant, and the Israeli I-Core program. 


\section{References}

1. Y. Afek, N. Alon, O. Barad, E. Hornstein, N. Barkai, and Z. Bar-Joseph. A biological solution to a fundamental distributed computing problem. Science, 331(6014):183-185, 2011.

2. N. Alon, L. Babai, and A. Itai. A fast and simple randomized parallel algorithm for the maximal independent set problem. J. of Algorithms, 1986.

3. B. Awerbuch, A. V. Goldberg, M. Luby, and S. A. Plotkin. Network decomposition and locality in distributed computation. In Proc. 30th Symposium on Foundations of Computer Science (FOCS), pages 364-369, 1989.

4. B. Chlebus, L. Gasieniec, A. Gibbons, A. Pelc, and W. Rytter. Deterministic broadcasting in unknown radio networks. In Prof. 11th Symposium on Discrete Algorithms (SODA), pages 861-870, 2000.

5. J.R. Collier, N.A. Monk, P.K. Maini, and J.H. Lewis. Pattern formation by lateral inhibition with feedback: a mathematical model of delta-notch intercellular signalling. J Theor Biol, 183(4):429-46, 1996.

6. A. Cornejo and F. Kuhn. Deploying wireless networks with beeps. In Proc. 24th Symposium on Distributed Computing (DISC), pages 148-162, 2010.

7. J. Degesys, I. Rose, A. Patel, and R. Nagpal. Desync: self-organizing desynchronization and TDMA on wireless sensor networks. In Prof. 6th Conf. on Information Processing in Sensor Networks (IPSN), page 20, 2007.

8. R. Flury and R. Wattenhofer. Slotted programming for sensor networks. Proc. 9th Conf. on Information Processing in Sensor Networks (IPSN), 2010.

9. D. Ilcinkas, D. Kowalski, and A. Pelc. Fast radio broadcasting with advice. Theoretical Computer Science, 411(14-15), 2010.

10. F. Kuhn, T. Moscibroda, and R. Wattenhofer. What cannot be computed locally! In Proc. 23rd Symposium on Principles of Distributed Computing (PODC), pages 300-309, 2004.

11. F. Kuhn, T. Moscibroda, T. Nieberg, and R. Wattenhofer. Fast deterministic distributed maximal independent set computation on growth-bounded graphs. In Proc. 19th Symposium on Distributed Computing (DISC), pages 273 - 287, 2005.

12. F. Kuhn, T. Moscibroda, and R. Wattenhofer. The price of being near-sighted. In Proc. 17th ACMSIAM Symposium on Discrete Algorithms (SODA), pages 980-989, 2006.

13. M. Luby. A simple parallel algorithm for the maximal independent set problem. SIAM J. on Computing, 15:1036-1053, 1986.
14. Y. Métivier, J. Michael Robson, N. SahebDjahromi, and A. Zemmari. An optimal bit complexity randomized distributed mis algorithm. Distributed Computing, pages 331-340, 2011.

15. T. Moscibroda and R. Wattenhofer. Efficient computation of maximal independent sets in structured multi-hop radio networks. Proc. 1st Conf. on Mobile Ad Hoc Sensor Systems (MASS), 2004.

16. T. Moscibroda and R. Wattenhofer. Maximal Independent Sets in Radio Networks. Proc. 24th Symposium on Principles of Distributed Computing (PODC), 2005.

17. A. Motskin, T. Roughgarden, P. Skraba, and L. Guibas. Lightweight coloring and desynchronization for networks. In Proc. 28th IEEE Conf. on Computer Communications (INFOCOM), 2009.

18. A. Panconesi and A. Srinivasan. On the complexity of distributed network decomposition. J. of Algorithms, 20(2):581-592, 1995.

19. D. Peleg. Distributed computing: a locality-sensitive approach. Society for Industrial and Applied Mathematics, Philadelphia, PA, USA, 2000.

20. C. Scheideler, A. Richa, and P. Santi. An $O(\log n)$ dominating set protocol for wireless ad-hoc networks under the physical interference model. Proc. 9th Symposium on Mobile Ad Hoc Networking and Computing (MOBIHOC), 2008.

21. J. Schneider and R. Wattenhofer. A Log-Star Maximal Independent Set Algorithm for GrowthBounded Graphs. In Proc. 28th Symposium on Principles of Distributed Computing (PODC), 2008.

22. J. Schneider and R. Wattenhofer. What is the use of collision detection (in wireless networks)? In Proc. 24th Symposium on Distributed Computing (DISC), 2010.

23. P.-J. Wan, K. M. Alzoubi, and O. Frieder. Distributed construction of connected dominating set in wireless ad hoc networks. Mobile Networks and Applications, 2004. 